\title{
Modelagem do Efeito Chicote em ambientes com demanda e lead time estocásticos mediante uma nova política de tratamento dos excessos de estoque
}

\author{
José Carlos Fioriolli UFRGS \\ Flávio Sanson Fogliatto UFRGS
}

\section{RESUMO}

Este artigo analisa o desempenho dos principais modelos de quantificação do Efeito Chicote (EC) a partir do estudo do impacto produzido por diferentes políticas de tratamento dos excessos de estoque sobre o EC, em ambientes com demanda e lead time estocásticos. Além da política considerada como pressuposto dos principais modelos de quantificação do EC disponíveis na literatura, onde excedentes de estoque são devolvidos a custo zero, outras duas políticas são investigadas: (i) excedentes são desconsiderados, ou seja, não motivam nem compra nem devolução de itens, e (ii) excedentes são ajustados e utilizados para suprir demandas futuras. A política (ii), considerada como a que melhor retrata a realidade operacional nas empresas, é incorporada à modelagem do EC sob a forma de um fator de correção em sua quantificação. Produz-se, assim, um novo modelo matemático para dimensionamento do EC em ambientes sujeitos à demanda e lead time estocásticos e independentes, onde excessos de estoque são incorporados no cálculo do tamanho dos pedidos futuros. 0 desenvolvimento deste novo modelo é apresentado detalhadamente, assim como as simulações computacionais realizadas para comparar seu desempenho com os modelos de Chen et al. (2000) e Fioriolli e Fogliatto (2007).

Efeito chicote, quantificação, modelagem estocástica, lead times estocásticos.

\section{Modeling the Bullwhip Effect under stochastic demand and lead time considering a new policy for inventory excesses}

\begin{abstract}
In this paper we analyze the performance of the main models developed to quantify the Bullwhip Effect (BE) focusing on the impact due to the adoption of different policies to treat inventory excesses, under the assumption of stochastic demand and lead time. In addition to the policy assumed in most BE quantification models available in the literature, where inventory excesses are handed back to suppliers at no cost, two alternative policies are investigated: (i) one where excesses are disregarded from the analysis, not motivating product devolution, and (ii) another where excesses are adjusted and used to supply for future demands. Policy (ii), considered as the one best reproducing the operational reality of companies, is incorporated to the BE modeling as a correction factor when quantifying the phenomenon. We thus present a new mathematical model to quantify the BE in situations where both demand and lead time are stochastic, and where inventory excesses are considered in the determination of future orders. The development of the new model is presented along with computational simulations run to compare its performance with models in Chen et al. (2000) and Fioriolli \& Fogliatto (2007).
\end{abstract}

KEY WORDS

Bullwhip effect, quantification, stochastic modeling, stochastic lead times. 


\section{INTRODUÇÃO}

O Efeito Chicote (EC) é um importante fenômeno que produz impactos negativos sobre a regularidade e a estabilidade dos pedidos recebidos em todos os níveis de uma cadeia de abastecimento. De acordo com Chen et al. (2003), observa-se um EC quando a variabilidade da demanda aumenta na medida em que se avança nos níveis da cadeia, desde o varejista até o fabricante.

Para avaliar adequadamente as implicações gerenciais do EC é importante entender a sua dinâmica, ilustrada na Figura 1. Considerando dois níveis adjacentes $k$ e $k-1 \mathrm{em}$ uma cadeia de abastecimento, esta dinâmica pode ser assim descrita. O pedido gerado pelo nível $k$ é definido a partir da previsão de demanda do nível anterior $(k-1)$ e depende dos dados históricos relativos àquela demanda e da técnica de previsão utilizada no nível $k$. Da mesma forma, os pedidos gerados pelo nível $k+1$ são definidos em função dos dados históricos disponíveis sobre a demanda do nível anterior $(k)$ e da técnica de previsão utilizada no nível $k+1$. Esta lógica se repete a cada avanço de nível, de modo que os dados disponíveis, em cada nível (com exceção do nível mais baixo da cadeia), baseiam-se em uma expectativa de demanda e não na demanda real.

conforme demonstrado por Anderson e Fine (2003); (iv) processo decisório reativo, principalmente em função dos picos de demanda, causando ruptura de planejamento; e $(v)$ gestão ineficiente dos recursos da cadeia de abastecimento como um todo, em decorrência das ineficiências locais e da dificuldade de integração das operações realizadas.

O principal desafio relativo ao gerenciamento do EC consiste em reduzir ineficiências e atenuar/eliminar a sua propagação ao longo da cadeia de abastecimento, de modo a viabilizar a otimização dos recursos empregados em seus diferentes níveis. Isto depende do grau de conhecimento que se tem sobre as suas causas e sobre a sua intensidade.

Nesse sentido, a quantificação do EC tem sido um tema freqüente de pesquisa nos últimos anos. Autores como Lee et al. (1997), Chen et al. (2000), Fransoo e Wouters (2000) e Warburton (2004) vêm apresentando importantes desenvolvimentos teóricos sobre o tema. Um dos principais modelos de quantificação do EC, apresentado por Chen et al. (2000), é formulado como função do lead time, da variância da demanda e do número de períodos utilizados na previsão da demanda. O modelo trabalha com cenários com lead time constante. De modo similar, os modelos de Lee et al. (1997), Fransoo e Wouters (2000) e Warburton (2004) também operam em ambientes com lead time constante. Tal restrição nesses modelos resulta em uma quantificação imprecisa do EC, sendo aplicáveis em cenários raramente encontrados na prática.

Fioriolli e Fogliatto (2007) propõem um modelo matemático para quantificação do EC

Conforme Lee et al. (1997), Carlsson e Fullér (2000) e Chen et al. (2003), o EC potencialmente traz consequências locais e sistêmicas para fabricantes, distribuidores e varejistas. Entre as consequências locais, destacam-se (i) baixos níveis de serviço, gerados pela dificuldade de amortecer, em tempo hábil, as variações extremas da demanda; (ii) vendas perdidas em função das rupturas de estoques geradas por variações extremas da demanda; (iii) aumentos dos estoques de segurança, com vistas à recuperação dos níveis de serviço que garantam a competitividade da estrutura; (iv) aumento no número de reprogramações de produção para cobrir emergências; e $(v)$ gestão ineficiente de recursos locais, como pessoal, equipamentos e capital. Entre as consequências sistêmicas, destacam-se: (i) elevação dos custos relacionados a estoques na cadeia de abastecimento, em razão do aumento dos estoques locais, em cada um dos pontos do sistema; (ii) queda do retorno sobre o capital investido nas operações da cadeia; (iii) queda da produtividade dos funcionários que atuam nos processos produtivos desenvolvidos no sistema, em ambientes que utilizam a política de pedidos do tipo out-EA (order-up-to Estoque Alvo), sujeitos à demanda e lead time estocásticos e independentes, supondo ambas as variáveis com distribuições de probabilidade que podem ser aproximadas por uma normal. Os autores demonstram que o modelo de Chen et al. (2000) é um caso especial do modelo por eles desenvolvido e que sua utilização resulta em uma quantificação mais precisa do EC. Entretanto, ambos os modelos, bem como aqueles propostos por Lee et al. (1997), Fransoo e Wouters (2000) e Warburton (2004), tratam eventuais valores negativos obtidos no cálculo do tamanho dos pedidos como sendo excessos de estoque, que podem ser devolvidos a custo zero. Tal suposição compromete a aplicabilidade prática dos modelos.

Este artigo analisa o impacto produzido por diferentes políticas de tratamento dos excessos de estoque sobre a quantificação do EC em ambientes com demanda estocástica. Além da política considerada como pressuposto dos modelos de quantificação do EC disponíveis na literatura, 
aqui denominada de política P1, onde excedentes de estoque são devolvidos a custo zero, outras duas políticas são propostas e investigadas: $\mathrm{P} 2$ - tamanhos de pedido negativos são considerados como nulos, ou seja, não motivam nem compra nem devolução de itens, e P3 - tamanhos de pedido negativos sinalizam estoques excedentes, os quais podem ser utilizados para suprir demandas futuras. A política P3 de tratamento dos excessos de estoque, considerada como a que melhor retrata a realidade operacional nas empresas, é incorporada ao modelo de Fioriolli e Fogliatto (2007) na forma de um fator de correção na quantificação do EC. Chega-se, assim, a um novo modelo matemático de quantificação do EC em ambientes sujeitos à demanda e lead time estocásticos e independentes, onde excessos de estoque são incorporados no cálculo do tamanho dos pedidos futuros.
O presente artigo apresenta três contribuições relevantes para o estudo do EC em cadeias de abastecimento. Primeiro, demonstra-se que o modelo mais referenciado de quantificação do EC, devido a Chen et al. (2000), mede corretamente o fenômeno em cenários bastante limitados de aplicação. Segundo, analisa-se em profundidade um dos pressupostos que regem o desenvolvimento e a utilização da maioria dos modelos de quantificação do EC disponíveis na literatura: o tratamento de excessos de estoque como devoluções sem custo. Os desenvolvimentos no artigo demonstram que, além de resultar em uma quantificação imprecisa do EC, tal pressuposto não representa a realidade da prática da gestão de cadeias de abastecimento. Para contornar essa deficiência, propõe-se uma política mais realista para o tratamento de excessos de estoques. Terceiro, propõe-se um novo modelo

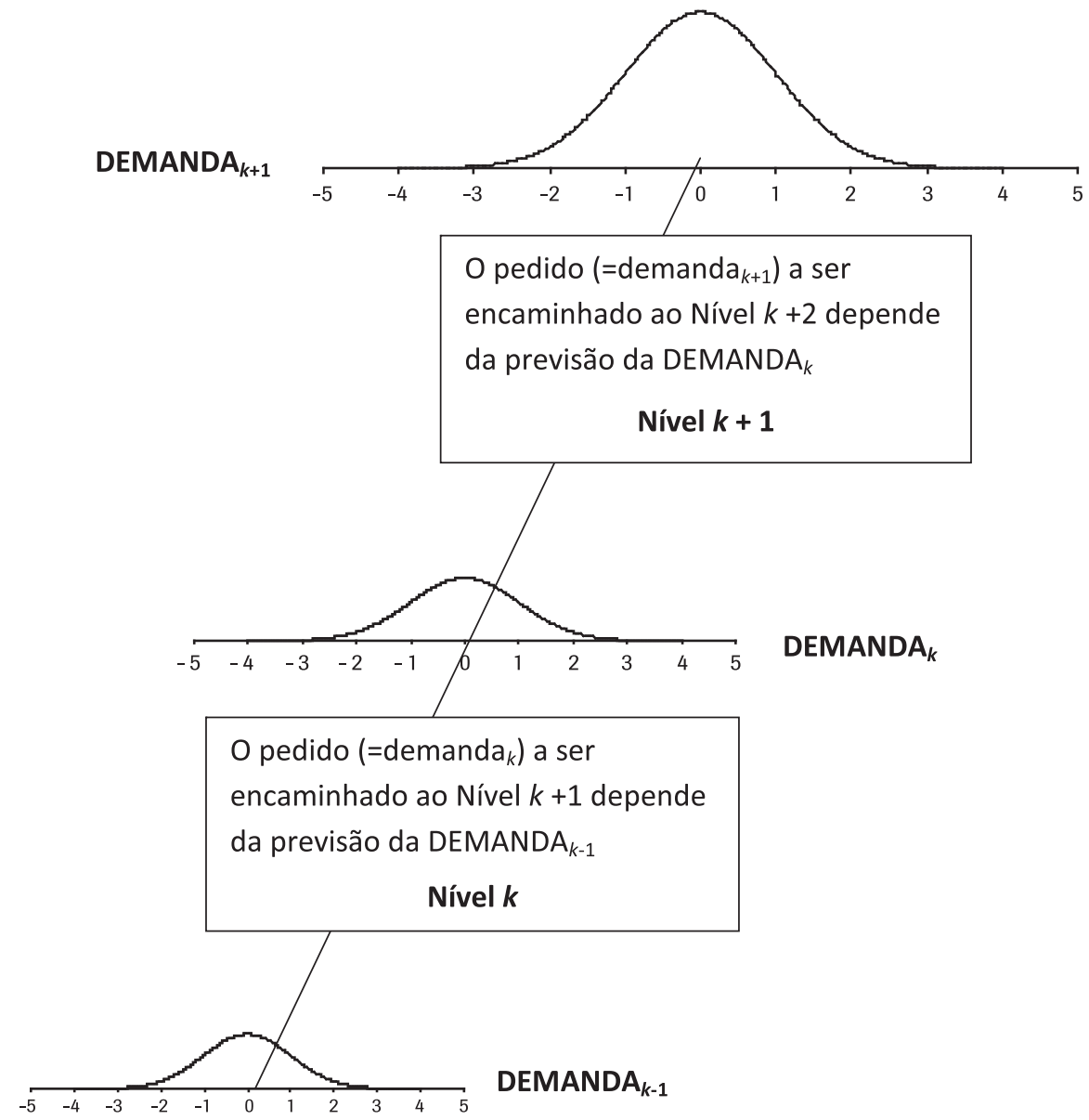

Figura 1: Dinâmica do EC.

Fonte: adaptada de Chen et al. (2003) 
matemático para quantificação do EC o qual, além de considerar a variabilidade nos lead times de entrega de pedidos nos diferentes níveis da cadeia de abastecimento, trata de modo mais adequado os excessos de estoque, incorporando os necessários ajustes no cálculo do tamanho dos pedidos.

O desenvolvimento de uma modelagem estocástica de contribuição original e relevante para o estudo do EC, já que permite uma maior precisão na identificação das causas deste fenômeno, facilitando o entendimento da sua dinâmica. Ao incorporar os necessários ajustes no cálculo do tamanho dos pedidos, o modelo retrata com maior realismo as situações observadas nas cadeias de abastecimento, tratando adequadamente os excessos de estoque. Isto é fundamental para a adoção de medidas gerenciais que visem à redução dos impactos negativos do EC sobre os estoques e os níveis de serviço, o que justifica, também na prática, os desenvolvimentos aqui propostos. acordo com a proposta aqui apresentada se configura em

(1997), ocorre uma distorção da demanda, que se propaga ao longo da cadeia de abastecimento através do fluxo básico de informação e produz uma amplificação da variabilidade dos pedidos encaminhados aos fornecedores.

Considerando uma estrutura com três níveis (loja, depósito e fornecedor), observa-se que a demanda dos clientes apresenta pequenas variações durante o horizonte de análise. Em razão da distorção da informação no sistema, os pedidos da loja para o depósito apresentam variações maiores em comparação à demanda dos clientes. No último nível, os pedidos encaminhados ao fornecedor estão amplificando as variações observadas na loja e no depósito.

As abordagens utilizadas para a quantificação do EC podem ser divididas em três grupos: ( $i$ ) cálculo da relação entre a variância dos pedidos e a variância da demanda, utilizado por Lee et al.(1997), Chen et al. (2000), Hosoda e Disney (2004) e Fioriolli e Fogliatto (2007); (ii) cálculo da relação entre a taxa de pedidos e a taxa da demanda, utilizado por Warburton (2004); e (iii) cálculo do quociente entre o coeficiente de variação da demanda gerada por um nível da cadeia e o coeficiente de variação da demanda recebida por este mesmo nível, utilizado por Fransoo e Wouters (2000).

A revisão que se segue limita-se aos modelos de Chen et al. (2000) e Fioriolli e Fogliatto (2007), os quais são diretamente

$\mathrm{O}$ restante deste artigo está organizado em quatro seções. Na seção 2, apresentam-se os modelos propostos por Chen et al. (2000) e Fioriolli e Fogliatto (2007). Na seção 3 , inicialmente apresentam-se três políticas de tratamento de excessos de estoque e seu efeito sobre a variabilidade do tamanho dos pedidos; na seqüência, definida a melhor política, esta é utilizada como base para o desenvolvimento de um novo modelo matemático para a quantificação do EC, o qual é então apresentado. Tal modelo estende a proposição em Fioriolli e Fogliatto (2007) para cenários onde excessos de estoque são incorporados no cálculo do tamanho dos pedidos futuros. O desempenho do modelo proposto é comparado com as proposições de Chen et al. (2000) e de Fioriolli e Fogliatto (2007) em cenários simulados. Uma conclusão encerra o artigo na seção 5.

\section{DOIS MODELOS PARA QUANTIFICAÇÃO DO EC}

O EC refere-se a uma situação em que o tamanho dos pedidos enviados aos fornecedores tende a apresentar maior variância do que as vendas efetuadas junto aos compradores (CARLSSON; FULLÉR, 2001). De acordo com Lee et al. relacionados aos desenvolvimentos neste artigo. As notações utilizadas nestes modelos foram uniformizadas, estando apresentadas na Tabela 1. Para manter a brevidade da exposição, recomendam-se as referências indicadas no parágrafo anterior para um detalhamento dos demais modelos de quantificação do EC.

Chen et al. (2000) consideram uma cadeia de abastecimento em que a cada período $t$ um varejista observa seu nível de estoque e coloca um pedido $Q_{t}$ para um fabricante. Após o pedido ter sido colocado, o varejista observa e atende a demanda $D_{t}$ relativa ao período em curso; demandas não atendidas ficam pendentes. Um lead time $L$, constante, transcorre entre o momento em que o pedido é colocado pelo varejista e o momento em que o pedido é recebido por ele, de modo que um pedido colocado no fim de um período $t$ é recebido no início do período $t+L$.

A demanda vista pelo varejista é uma variável aleatória, cujo processo de formação é representado por um modelo autoregressivo de primeira ordem:

$$
D_{t}=d+\rho D_{t-1}+\varepsilon_{t}
$$

O varejista segue uma política order-up-to em que o 
estoque-alvo é estimado a partir da demanda observada durante o lead time e assim definido:

$$
A_{t}=\hat{D}_{L, t}+z \hat{\sigma}_{e, t}^{L}
$$

onde $\hat{D}_{L, t}=L\left(\sum_{i=1}^{p} D_{t-1} / p\right)$ e $\hat{\sigma}_{e, t}^{L}=C_{L, p}\left(\sum_{i=1}^{p}\left(e_{t-i}\right)^{2} / p\right)^{1 / 2}$. O pedido é calculado em função do estoque-alvo e da demanda, sendo dado na eq. (3). Eventuais valores negativos são assumidos como excessos de estoque que podem ser devolvidos sem custo, conforme proposto por Kahn (1987) e Lee et al. (1997).

$$
Q_{t}=A_{t}-A_{t-1}+D_{t-1}
$$

O cálculo do pedido pode ser apresentado em função das estimativas da demanda durante o lead time, da constante vinculada ao nível de serviço desejado, do desvio-padrão dos erros de previsão relativos ao mesmo período e da demanda observada no período anterior.

$$
Q_{t}=(1+L / p) D_{t-1}-(L / p) D_{t-p-1}+z\left(\hat{\sigma}_{e, t}^{L}-\hat{\sigma}_{e, t-1}^{L}\right)
$$

Pela definição do EC, segue que:

$$
E C=\frac{\operatorname{Var}(Q)}{\operatorname{Var}(D)} \geq 1+\left(\frac{2 L}{p}+\frac{2 L^{2}}{p^{2}}\right)\left(1-\rho^{p}\right)
$$

No caso específico de não existir correlação entre as demandas período a período, ou seja, quando $\rho=0$ :

$$
E C=\frac{\operatorname{Var}(Q)}{\operatorname{Var}(D)} \geq 1+\frac{2 L}{p}+\frac{2 L^{2}}{p^{2}}
$$

\begin{tabular}{|c|c|}
\hline Símbolo & Descrição \\
\hline$t$ & Subscrito que denota o momento de observação da variável no tempo \\
\hline$L$ & Lead time constante \\
\hline$p$ & Número de períodos utilizados no cálculo da média móvel da demanda \\
\hline$d$ & Constante não negativa, utilizada no modelo auto-regressivo do processo de formação da demanda \\
\hline$\rho$ & Parâmetro de correlação do processo de formação da demanda \\
\hline$\varepsilon_{t}$ & Erro aleatório em $t$, normalmente distribuído, com média 0 e desvio-padrão $\sigma$ \\
\hline$D_{t}$ & Demanda estocástica em $t$ \\
\hline$\hat{D}_{L, t}$ & Estimativa da demanda média durante o lead time, em $t$ \\
\hline$e_{t}$ & Erro de previsão da demanda em $t$ \\
\hline$C_{L, p}$ & Função constante de $L, \rho$ e $p$ \\
\hline$\hat{\sigma}_{e, t}^{L}$ & Estimativa do desvio-padrão do erro de previsão de demanda durante o lead time, em $t$ \\
\hline$z$ & Constante correspondente ao nível de serviço desejado \\
\hline$A_{t}$ & Estoque-alvo (order-up-to) em $t$ \\
\hline$Q_{t}$ & Tamanho do pedido em $t$ \\
\hline$k$ & Nível hierárquico da estrutura em análise \\
\hline$\mu_{D}$ & Média da demanda \\
\hline$\sigma_{D}^{2}, \sigma_{D}$ & Variância e desvio-padrão da demanda \\
\hline$\theta_{D}$ & Coeficiente de variação da demanda \\
\hline$\mu_{L}$ & Média do lead time \\
\hline$\sigma_{L}^{2}, \sigma_{L}$ & Variância e desvio-padrão do lead time \\
\hline$L_{t}$ & Lead time em $t$ \\
\hline$\hat{D}_{t}$ & Estimativa da demanda em $t$ \\
\hline
\end{tabular}

Conforme exposto acima, o modelo de quantificação do EC na eq. (5) trabalha com cenários onde o lead time é

Tabela 1: Notação utilizada nos modelos do referencial teórico. 
constante. Fioriolli e Fogliatto (2007) propõem um modelo matemático para quantificação do EC baseado ( $i$ ) na independização do processo de formação da demanda, e (ii) na incorporação da variabilidade do lead time.

Na representação da demanda, a maioria dos autores trabalha com modelos autoregressivos de primeira ordem. Lee et al. (1997), Chen et al. (2000) e Warburton (2004) utilizam o mesmo modelo de formação da demanda apresentado na cadeia de abastecimento em que, a cada período $t$, um varejista avalia seu nível de estoque e envia um pedido $Q_{t}$ para um fabricante, a exemplo do trabalho de Chen et al. (2000). Após o pedido ter sido encaminhado, o varejista observa e atende (caso o estoque seja suficiente) a demanda $D_{t}$ relativa ao período em curso. Eventuais demandas não atendidas ficam pendentes. Considera-se a ocorrência de um lead time variável $L_{t}$ entre o momento em que o pedido é encaminhado pelo varejista e o momento em que o pedido é recebido por ele. Um pedido colocado no fim do período $t$ é recebido no início do período $t+L_{t}$. A notação utilizada no modelo vem apresentada na Tabela 1 .

A demanda vista pelo varejista e o lead time de entrega dos pedidos são variáveis aleatórias, normalmente distribuídas,

eq. (1). Rajaram e Robotis (2002), por sua vez, utilizam um modelo linear de primeira ordem para capturar a propagação da variabilidade da demanda. Fioriolli e Fogliatto (2007) baseiam seu modelo na proposição de Kuncová (2002), segundo a qual é possível modelar o EC trabalhando com o pressuposto de que a demanda é uma variável aleatória que pode ser aproximada por uma distribuição normal, sem a necessidade de modelos preliminares que descrevam a sua formação. Tal suposição também está na base do desenvolvimento dos modelos clássicos de dimensionamento do lote de compra, como apresentado em Elsayed e Boucher (1994) e Silver et al. (1998).

No que diz respeito à incorporação da variabilidade do lead time, Fioriolli e Fogliatto (2007) trabalham com o pressuposto de que o lead time pode ser representado por uma distribuição normal. De acordo com Silver et al. (1998), tais pressupostos simplificadores são razoáveis na medida em que conseguem representar adequadamente a realidade em situações em que o uso de abordagens mais realistas torna os modelos finais demasiadamente complexos e, por isso, pouco úteis em termos práticos. Nesta incorporação, Fioriolli e Fogliatto (2007) desenvolveram os seguintes procedimentos: (a) introdução do desvio padrão do lead time no cálculo da variabilidade da demanda estimada para o período correspondente ao lead time e, por consequência, no cálculo dos estoques-alvo desejados; (b) quantificação da influência que o lead time variável e o número de períodos (usado no cálculo da média da demanda) exercem conjuntamente sobre a variabilidade da estimativa da demanda durante o lead time e também sobre o tamanho do pedido; e $(c)$ identificação da correlação entre os estoques-alvo calculados e entre estes e a demanda.

O modelo em Fioriolli e Fogliatto (2007) considera uma com média e desvio-padrão conhecidos ou estimáveis a partir de dados históricos; isto é, $D \rightarrow N\left(\mu_{D}, \sigma_{D}\right)$ e $L \rightarrow N\left(\mu_{L}, \sigma_{L}\right)$. A estimativa da demanda em $t$ é calculada a partir de uma média móvel que utiliza os dados relativos à demanda observada nos últimos $p$ períodos:

$$
\hat{D}_{t}=\sum_{i=1}^{p} D_{t-i} / p
$$

De acordo com Mood et al. (1974, p. 231), a demanda estimada através da eq. (7) pode ser representada por uma distribuição normal, tal que $\hat{D} \rightarrow N\left(\mu_{D}, \sigma_{D} / p^{0,5}\right)$. A estimativa da demanda durante o lead time é obtida utilizando a média móvel referida na eq. (7) e o lead time em $t$, a partir do pressuposto que $L_{t}$ e $\hat{D}_{t}$ utilizam a mesma unidade de tempo.

$$
\hat{D}_{L, t}=L_{t} \hat{D}_{t}=L_{t}\left(\sum_{i=1}^{p} D_{t-1} / p\right)
$$

A variância do produto de duas variáveis aleatórias independentes, tais como demanda e lead time, é expressa como uma soma de três parcelas: (i) o produto da variância da primeira variável com o quadrado da média da segunda variável; (ii) o produto da variância da segunda variável com o quadrado da média da primeira variável; e (iii) o produto das variâncias das duas variáveis (MOOD et al., 1974, p. 180). Segundo esses mesmos autores, a expectância do produto de duas variáveis independentes é igual ao produto das médias das variáveis. Com base nestas definições e nas suposições acima apresentadas, a estimativa da demanda no lead time pode ser representada por uma distribuição normal, com média e desvio-padrão assim expressos:

$$
\hat{D}_{L} \rightarrow N\left(\mu_{D} \mu_{L},\left(\sigma_{L}^{2} \mu_{D}^{2}+\left(\sigma_{D}^{2} / p\right)\left(\mu_{L}^{2}+\sigma_{L}^{2}\right)\right)^{0,5}\right) .
$$


O varejista segue uma política order-up-to em que o estoque-alvo é calculado a partir da estimativa da demanda durante o lead time, do nível de segurança desejado e do desvio-padrão da demanda estimada para o lead time:

$$
A_{t}=\hat{D}_{L, t}+z \hat{\sigma}_{D L, t},
$$

onde $\hat{D}_{L, t}=L_{t}\left(\sum_{i=1}^{p} D_{t-i} / p\right) \mathrm{e}$

$\hat{\sigma}_{D L, t}=\left(\sigma_{L}^{2} \mu_{D}^{2}+\left(\sigma_{D}^{2} / p\right)\left(\mu_{L}^{2}+\sigma_{L}^{2}\right)\right)^{0,5}$. O pedido é calculado em função do estoque-alvo e da demanda. Eventuais valores negativos são assumidos como excessos de estoque que podem ser devolvidos sem custo, de acordo com Kahn (1987), Lee et al. (1997) e Chen et al. (2000).

$$
Q_{t}=A_{t}-A_{t-1}+D_{t-1}=\left(\hat{D}_{L, t}+z \hat{\sigma}_{D L, t}\right)-\left(\hat{D}_{L, t-1}+z \hat{\sigma}_{D L, t-1}\right)+D_{t-1}
$$

O cálculo do pedido pode ser apresentado em função da diferença entre as estimativas da demanda durante o lead time (períodos $t$ e $t-1$ ), da constante vinculada ao nível de serviço desejado, da diferença entre as estimativas do desviopadrão da demanda estimada para o lead time (períodos $t \mathrm{e}$ $t-1)$ e da demanda observada no período $t-1$.

$$
\begin{gathered}
Q_{t}=L_{t}\left(\sum_{i=1}^{p} D_{t-i} / p\right)-L_{t-1}\left(\sum_{i=1}^{p} D_{t-i-1} / p\right) \\
+z\left(\hat{\sigma}_{D L, t}-\hat{\sigma}_{D L, t-1}\right)+D_{t-1}
\end{gathered}
$$

A Tabela 2 traz definições adicionais, a serem utilizadas nos desenvolvimentos que se seguem.

Tabela 2: Definições adicionais.

$$
\begin{gathered}
E_{1}=L_{t}\left(\left(\sum_{i=1}^{p} D_{t-i}\right) / p\right) \\
E_{2}=L_{t-1}\left(\left(\sum_{i=1}^{p} D_{t-i-1}\right) / p\right) \\
E=E_{1}-E_{2} \\
F=z\left(\hat{\sigma}_{D L, t}-\hat{\sigma}_{D L, t-1}\right) \\
G=D_{t-1} \\
Q_{t}=E+F+G
\end{gathered}
$$

Conforme Mood et al. (1974, p. 178), ao calcular a variância de uma soma de variáveis aleatórias correlacionadas, deve-se somar as variâncias de cada uma das variáveis e adicionar, a este subtotal, o dobro das covariâncias relativas a cada par de variáveis. Portanto, a variância do tamanho dos pedidos deve ser calculada da seguinte forma:

$$
\operatorname{Var}_{Q_{t}}=\operatorname{Var}_{E}+\operatorname{Var}_{F}+\operatorname{Var}_{G}+2\left(\operatorname{Cov}_{E F}+\operatorname{Cov}_{E G}+\operatorname{Cov}_{F G}\right)
$$

Dado que $E=E_{1}-E_{2}$, torna-se necessário calcular a variância desta diferença. Como as variáveis $E_{1}$ e $E_{2}$ estão correlacionadas, tem-se (MOOD et al., 1974, p. 179):

$$
\begin{gathered}
\operatorname{Var}_{E}=\operatorname{Var}_{E_{1}}+\operatorname{Var}_{E_{2}}-2 \operatorname{Cov}_{E_{1} E_{2}} \\
\operatorname{Var}_{E_{1}}=\operatorname{Var}_{E_{2}}=\sigma_{L}^{2} \mu_{D}^{2}+\sigma_{D}^{2} / p\left(\mu_{L}^{2}+\sigma_{L}^{2}\right)
\end{gathered}
$$

A covariância entre $E_{1}$ e $E_{2}$ foi modelada matematicamente por Fioriolli e Fogliatto (2007) a partir de um conjunto de simulações computacionais. Os autores trabalharam com diversos cenários, considerados como viáveis na prática, combinando três valores de coeficiente de variação da demanda $(0,1 ; 1$ e 2$)$, três valores de coeficiente de variação do lead time $(0,1 ; 1$ e 2$)$ e três valores de $p(2 ; 10$ e 30$)$. Para cada um dos 27 cenários, foram realizadas 5000 rodadas da rotina de cálculo da covariância entre $E_{1}$ e $E_{2}$, conforme as eqs. (13) e (14). Em cada rodada, foi utilizada uma série de 2000 valores de demanda e lead time, gerados aleatoriamente. A covariância entre $E_{1}$ e $E_{2}$ foi determinada, por prova sintética, como:

$$
\operatorname{Cov}_{E_{1} E_{2}}=\sigma_{D}^{2}\left((p-1) / p^{2}\right) \mu_{L}^{2}
$$

Utilizando-se a eq. (22), é possível determinar a variância da diferença entre as estimativas da demanda durante o lead time:

$$
\operatorname{Var}_{E}=2\left(\sigma_{L}^{2} \mu_{D}^{2}+\sigma_{D}^{2}\left(\mu_{L}^{2} / p^{2}+\sigma_{L}^{2} / p\right)\right)
$$

Na sequência, de acordo com a eq. (19), deve-se calcular a variância da diferença entre as estimativas do desvio-padrão da demanda durante o lead time, a variância da demanda em $t$ e as covariâncias entre (i) $E$ e $F$, (ii) $E$ e $G$ e (iii) $F$ e $G$.

A variância da diferença entre as estimativas do desviopadrão (em $t$ e $t-1$ ) foi modelada matematicamente por Fioriolli e Fogliatto (2007) através de uma série de simulações computacionais. Os autores trabalharam com um conjunto de cenários considerados como viáveis, combinando quatro valores de coeficiente de variação da demanda $(0,1$; 0,5 ; 1 e 2 ) e três valores de $z$ ( $1 ; 2$ e 3 ). Para cada um dos 12 cenários, foram realizadas 1000 rodadas da rotina de cálculo da variância, dada na eq. (16). Em cada rodada, os 
autores utilizaram uma série de 2000 valores de demanda gerados aleatoriamente. A variância da diferença entre as estimativas do desvio-padrão (em $t$ e $t-1$ ) foi modelada conforme a expressão proposta na eq. (24), obtida por prova sintética.

$$
\operatorname{Var}_{F}=2 z^{2}\left((t-1) / 3(t+1)^{2}\right) \sigma_{D L}^{2}
$$

Representando o fator dependente do tempo por $T$ e calculando a variância da demanda durante o lead time como $\sigma_{D L}^{2}=\sigma_{L}^{2} \mu_{D}^{2}+\left(\sigma_{D}^{2} / p\right)\left(\mu_{L}^{2}+\sigma_{L}^{2}\right)$, de acordo com Mood et al. (1974, p. 180-231), tem-se:

$$
\operatorname{Var}_{F}=2 z^{2} T\left(\sigma_{L}^{2} \mu_{D}^{2}+\left(\sigma_{D}^{2} / p\right)\left(\mu_{L}^{2}+\sigma_{L}^{2}\right)\right) .
$$

Na seqüência, calcula-se a variância de $G$, conforme a eq. (17):

$$
\operatorname{Var}_{G}=\sigma_{D}^{2} .
$$

Obtidas as variâncias de $E, F$ e $G$ [eqs. (23), (25) e (26), respectivamente], calculam-se as covariâncias dessas variáveis. Fioriolli e Fogliatto (2007) verificaram, por simulação, que as covariâncias entre $E$ e $F$ e entre $F$ e $G$ convergem rapidamente para zero.

A covariância entre $E$ e $G$ também foi modelada matematicamente por Fioriolli e Fogliatto (2007). A partir de um conjunto de simulações computacionais, os autores trabalharam com diversos cenários, combinando três valores de coeficiente de variação da demanda $(0,1 ; 1$ e 2$)$, três valores de coeficiente de variação do lead time $(0,1$; 1 e 2$)$ e três valores de $p(2 ; 10$ e 30$)$. Para cada um dos 27 cenários, foram realizadas 5000 rodadas da rotina de cálculo da covariância entre $E$ e $G$, conforme as eqs. (15) e (17). Uma série de 2000 valores aleatórios de demanda e lead time foi utilizada pelos autores, em cada rodada. A expressão ajustada para $\operatorname{Cov}_{E G}$ é dada na eq. (27), tendo sido obtida por prova sintética.

$$
\operatorname{Cov}_{E G}=\sigma_{D}^{2} \mu_{L} / p
$$

Substituindo as parcelas da eq. (19) pelas respectivas expressões [eqs. (23), (25), (26) e (27)], tem-se:

$$
\begin{aligned}
\operatorname{Var}_{Q_{t}} & =\sigma_{D}^{2}\left[1+\frac{2 \mu_{L}}{p}\left(1+\frac{\mu_{L}}{p}\right)+\frac{2 \mu_{L}^{2} z^{2} T}{p}\right. \\
& \left.+2 \sigma_{L}^{2}\left(\frac{1}{\theta_{D}^{2}}+\frac{1}{p}\right)\left(1+z^{2} T\right)\right]
\end{aligned}
$$

Pela definição do EC $(E C=\operatorname{Var}(Q) / \operatorname{Var}(D))$, segue que:

$$
E C_{t}=1+\frac{2 \mu_{L}}{p}\left(1+\frac{\mu_{L}}{p}\right)+\frac{2 \mu_{L}^{2} z^{2} T}{p}+2 \sigma_{L}^{2}\left(\frac{1}{\theta_{D}^{2}}+\frac{1}{p}\right)\left(1+z^{2} T\right)
$$

Observa-se que o EC depende das seguintes variáveis: demanda, lead time, número de períodos utilizados no cálculo da média móvel da demanda, constante $z$ (correspondente ao nível de serviço desejado), variância da demanda, variância do lead time e tempo. Considerando a existência de $k$ níveis na CA, a quantificação do EC é modelada da seguinte maneira:

$$
\begin{gathered}
E C_{k t}=\prod_{j=1}^{k}\left(1+\frac{2 \mu_{L j}}{p_{j}}\left(1+\frac{\mu_{L j}}{p_{j}}\right)+\frac{2 \mu_{L j}^{2} z_{j}^{2} T}{p_{j}}\right. \\
\left.+2 \sigma_{L j}^{2}\left(\frac{1}{\theta_{D j}^{2}}+\frac{1}{p_{j}}\right)\left(1+z_{j}^{2} T\right)\right) \forall k
\end{gathered}
$$

Ao aplicar o modelo da eq. (29) à situação simplificada de lead time constante, tem-se:

$$
E C_{t}=1+\frac{2 L}{p}\left(1+\frac{L}{p}\right)+\frac{2 L^{2} z^{2} T}{p}
$$

Adicionalmente, considerando o valor de $z$ igual a zero (caso em que não se utiliza estoque de segurança), o modelo adquire a forma proposta por Chen et al. (2000), conforme representado na eq. (6).

$$
E C=1+\frac{2 L}{p}\left(1+\frac{L}{p}\right)=1+\frac{2 L}{p}+\frac{2 L^{2}}{p^{2}}
$$

\section{MODELO PROPOSTO}

O valor correspondente ao tamanho do pedido, calculado em função do estoque-alvo e da demanda, de acordo com a eq. (3), pode ser negativo; neste caso, o resultado é assumido como excesso de estoque, que pode ser devolvido sem custos, segundo Kahn (1987), Lee et al. (1997) e Chen et al. (2000). Em ambientes com lead time constante, esses valores negativos são eventuais e, de acordo com Chen et al. (2000), não impactam significativamente na intensidade do EC. Entretanto, a análise desses impactos em cenários diferentes daqueles utilizados por esses autores mostra que a frequência de tamanhos de pedido negativos é suficientemente alta para produzir distorções no dimensionamento do EC, mesmo em ambientes com lead time constante. A magnitude dessas 
distorções pode ser estimada a partir da variação observada na média e no desvio-padrão dos pedidos, conforme apresentado na Tabela 3 (dados gerados através de simulação, para fins de exemplo).

Os valores simulados referem-se às três políticas de tratamento dos excessos de estoque e permitem identificar os respectivos impactos sobre o EC: (i) devolução dos excessos de estoque sem custo (política P1); (ii) desconsideração dos excessos de estoque (política P2); e (iii) aproveitamento dos excessos de estoque no cálculo do tamanho dos pedidos dos períodos seguintes (política P3). Dependendo da política escolhida, o pedido ajustado poderá assumir valores diferentes do pedido original.

Ao adotar a política $\mathrm{P} 1$, o tamanho do pedido permanece inalterado, independentemente de seu valor ser positivo ou negativo. Desta forma, o pedido ajustado $Q_{t}^{R}$ é igual ao pedido original:

$$
Q_{t}^{R}=Q_{t}
$$

Caso a política adotada seja P2, o tamanho do pedido será ajustado (para zero) somente se apresentar valores negativos:

$$
Q_{t}^{R}=\max \left\{0 ; Q_{t}\right\}
$$

Na política P3, o ajuste no tamanho do pedido dependerá do estoque em excesso, $H_{t}$, como segue.

$$
\begin{gathered}
H_{t}=\operatorname{abs}\left[\min \left\{0 ; Q_{t}-H_{t-1}\right\}\right] \\
Q_{t}^{R}=\max \left\{0 ; Q_{t}-H_{t-1}\right\}
\end{gathered}
$$

Diferentes tratamentos dos excessos de estoque produ- zem diferentes resultados no cálculo do tamanho dos pedidos, conforme a Tabela 3. Dada a possibilidade de ocorrência frequente de valores negativos para o tamanho dos pedidos, a política P1 deixa de ser realista. Nestas condições, o desviopadrão dos pedidos tende a aumentar expressivamente, $\mathrm{o}$ que distorce a quantificação do EC. No caso da política P2, ocorre um aumento expressivo do tamanho médio dos pedidos, pois os valores negativos são totalmente desprezados; esta situação também é pouco realista, uma vez que o total de pedidos, ao longo do tempo, é muito superior às reais necessidades expressas pela demanda (excesso de $25 \%$ no tamanho médio dos pedidos, no exemplo apresentado).

A política $\mathrm{P} 3$, além de manter a média dos pedidos em níveis adequados à demanda e aos estoques-alvo desejados, não inflaciona o desvio-padrão dos pedidos. Todos os excessos de estoque, na política P3, são considerados no cálculo do tamanho dos pedidos seguintes. Com a adoção da política P3, o ajuste realizado nos pedidos em função dos excessos de estoque reduz o desvio-padrão da série original de pedidos, sem alterar a sua média, conforme exemplo apresentado na Tabela 3. Desta forma, optou-se por incorporar a política P3 ao modelo proposto por Fioriolli e Fogliatto (2007). Os desenvolvimentos resultantes desta incorporação vêm apresentados na sequência.

Considere o modelo de quantificação do EC apresentado na eq. (29). Visando representar com maior realismo o tratamento dos estoques, todo e qualquer valor negativo de tamanho de pedido (considerado como um excesso de estoque $H_{t}$ ) pode ser utilizado no ajuste do tamanho do pedido, de acordo com as equações (35) e (36).

Este ajuste, realizado em função dos excessos de estoque,

\begin{tabular}{|c|c|c|c|c|c|c|c|c|c|}
\hline \multirow[b]{2}{*}{$t$} & \multicolumn{2}{|c|}{ Estoque-alvo } & \multirow{2}{*}{$\frac{\text { Demanda }}{D_{t-1}}$} & \multirow{2}{*}{$\frac{\text { Pedido }}{Q_{t}}$} & \multicolumn{2}{|c|}{ Excesso de estoque } & \multicolumn{3}{|c|}{ Pedido ajustado } \\
\hline & $A_{t}$ & $A_{t-1}$ & & & $H_{t-1}$ & $H_{t}$ & $\begin{array}{l}Q_{t}^{R} \\
\mathrm{P} 1\end{array}$ & $\begin{array}{l}Q_{t}^{R} \\
\mathrm{P} 2\end{array}$ & $\begin{array}{l}Q_{t}^{R} \\
\mathrm{P} 3\end{array}$ \\
\hline 1 & 160 & - & - & 160 & - & 0 & 160 & 160 & 160 \\
\hline 2 & 140 & 160 & 15 & -5 & 0 & 5 & -5 & 0 & 0 \\
\hline 3 & 200 & 140 & 45 & 105 & 5 & 0 & 105 & 105 & 100 \\
\hline 4 & 80 & 200 & 50 & -70 & 0 & 70 & -70 & 0 & 0 \\
\hline 5 & 100 & 80 & 30 & 50 & 70 & 20 & 50 & 50 & 0 \\
\hline 6 & 110 & 100 & 50 & 60 & 20 & 0 & 60 & 60 & 40 \\
\hline Média & 131,6 & - & 38 & 50 & - & 15,8 & 50 & 62,5 & 50 \\
\hline Desvio-padrão & 44,0 & - & 15,2 & 80,8 & - & 27,6 & 80,8 & 62,1 & 66,6 \\
\hline
\end{tabular}
altera somente o desvio-padrão da série original de pedidos. Desta forma, o novo coeficiente de variação dos pedidos, $\theta_{Q^{R}}$,

Tabela 3: Políticas de tratamento dos excessos de estoque. 
pode ser expresso como:

$$
\theta_{Q^{R}}=M \theta_{Q}
$$

O fator de ajuste do coeficiente de variação, $M$, pode ser estimado através da expressão proposta na Equação (38). A respectiva modelagem matemática foi desenvolvida a partir de um conjunto de simulações computacionais. Trabalhouse com um total de 29 cenários, elaborados com base em diferentes coeficientes de variação de pedidos. Para cada um dos cenários definidos, foram realizadas 1000 rodadas da rotina de cálculo do fator de ajuste. Utilizou-se, em cada rodada, uma série de 10000 valores gerados aleatoriamente.

$$
\hat{M}=\left[1-\mathrm{e}^{-a \theta_{Q}^{b}}\right]
$$

O desvio-padrão do pedido ajustado pode ser expresso por:

$$
\sigma_{Q^{R}}=\hat{M} \sigma_{Q}
$$

Pela definição do EC, segue que:

$$
\begin{gathered}
E C^{R}=\frac{\operatorname{Var}\left(Q^{R}\right)}{\operatorname{Var}(D)}=\hat{M}^{2} \frac{\operatorname{Var}(Q)}{\operatorname{Var}(D)}=\hat{M}^{2} E C \\
E C^{R}=\left[1-\mathrm{e}^{-a \theta_{Q}^{b}}\right]^{2} E C
\end{gathered}
$$

Como

$$
\begin{gathered}
\theta_{Q}=\frac{\sigma_{Q}}{\mu_{Q}} \\
\sigma_{Q}=\sigma_{D}\left[1+\frac{2 \mu_{L}}{p}\left(1+\frac{\mu_{L}}{p}\right)\right. \\
\left.+2 \sigma_{L}^{2}\left(\frac{1}{\theta_{D}^{2}}+\frac{1}{p}\right)\left(1+z^{2} T\right)+\frac{2 \mu_{L}^{2}}{p} z^{2} T\right]^{0,5}
\end{gathered}
$$

e, considerando $\mu_{Q}=\mu_{D}$, tem-se:

$$
\theta_{Q}=\theta_{D} E C_{t}^{0,5}
$$

Utilizando a eq. (44), pode-se reescrever a eq. (41) para definir a forma primária do modelo matemático proposto para a quantificação do EC:

$$
E C_{t}^{R}=\left[1-\mathrm{e}^{-a \theta_{D}^{b} E C_{t}^{0,5 b}}\right]^{2} E C_{t}
$$

sendo $a=2, b=-1 \mathrm{e}$ :

$$
\begin{gathered}
E C_{t}=\left[1+\frac{2 \mu_{L}}{p}\left(1+\frac{\mu_{L}}{p}\right)\right. \\
\left.+2 \sigma_{L}^{2}\left(\frac{1}{\theta_{D}^{2}}+\frac{1}{p}\right)\left(1+z^{2} T\right)+\frac{2 \mu_{L}^{2}}{p} z^{2} T\right]
\end{gathered}
$$

Considerando a existência de $k$ níveis na cadeia de abastecimento, a modelagem proposta para a quantificação do EC tem a seguinte forma:

$$
E C_{k}^{R}=\frac{\operatorname{Var}\left(Q_{k}^{R}\right)}{\operatorname{Var}\left(D_{1}\right)}=\prod_{j=1}^{k} E C_{j}^{R} \quad \forall k
$$

$$
E C_{k t}^{R}=\prod_{j=1}^{k}\left[1-\mathrm{e}^{-a \theta_{D j}^{b} E C_{j t}^{0,5 b}}\right]^{2} E C_{j t} \quad \forall k
$$

\section{ANÁLISE COMPARATIVA ENTRE MODELOS}

Na comparação entre os modelos utiliza-se um conjunto de resultados obtidos através de: $(i)$ simulações do EC, realizadas de acordo com o pressuposto de que eventuais valores negativos obtidos no cálculo do tamanho do pedido são assumidos como excessos de estoque, adotando as políticas P1 (onde os excessos são devolvidos sem custo) e P3 (onde os excessos são aproveitados nos períodos seguintes); e (ii) aplicações dos modelos [CHEN et al. (2000), FIORIOLLI; FOGLIATTO (2007) e a modelagem proposta nesta pesquisa]. A política P2 (onde os excessos de estoque são desconsiderados) não é utilizada na análise em razão das graves distorções provocadas sobre o total de pedidos, ao longo do tempo.

Inicialmente, trabalhou-se na identificação das variáveis (média e desvio-padrão da demanda, média e desvio-padrão do lead time, constante correspondente ao nível de serviço desejado e número de períodos) e dos parâmetros a serem utilizados nas simulações. Estas simulações, assim como todas as demais apresentadas nesta seção, estão disponíveis no endereço http://rapidshare.com/files/152105374/ SIMULACAO_MODELOS.xls. Os procedimentos foram desenvolvidos utilizando planilhas de cálculo em Excel $^{\circ}$ (com add-in da Crystal Ball ${ }^{\circ}$, disponível no endereço http:// portals.crystalball.com/Downloads).

Diversos cenários foram considerados, de acordo com as combinações obtidas a partir dos valores da Tabela 4 . 
Tabela 4: Variáveis e valores utilizados nas simulações.

\begin{tabular}{ccccc}
\hline$\mu_{D}$ & $\sigma_{D}$ & $\mu_{L}$ & $\sigma_{L}$ & $p$ \\
\hline 100 & 60 & 4 & 0 & 16 \\
200 & 80 & 6 & 1 & 18 \\
300 & 100 & 8 & 2 & 20 \\
\hline
\end{tabular}

Para cada um dos 243 cenários foram realizadas 2000 rodadas das rotinas de simulação e cálculo do EC. Utilizouse, em cada rodada, uma série de 1200 valores de demanda, gerados aleatoriamente. Os resultados obtidos indicam que há expressivas diferenças de desempenho entre os três modelos analisados.

As primeiras simulações foram desenvolvidas considerando ambientes com lead time estocástico; seus resultados constituem a base da análise comparativa sobre o desempenho dos modelos referidos anteriormente. Observa-se nas Figuras 2 e 3 que o modelo de Chen et al. (2000) tende a subestimar o EC (a exemplo do que é apresentado na Figura 3 , onde consegue quantificar apenas $5 \%$ da intensidade real do fenômeno), independentemente da política de tratamento de excessos de estoque utilizada (P1 ou P3). Isto ocorre porque a variabilidade do lead time não é considerada nesse modelo, apesar do expressivo impacto que produz sobre a intensidade do EC.

O modelo de Fioriolli e Fogliatto (2007) dimensiona adequadamente o EC quando a Política P1 é adotada (Figura 2), porém tende a superestimá-lo quando P3 é adotada (Figura 3). Essas superestimativas ocorrem porque o modelo, embora considere formalmente a variabilidade do lead time,

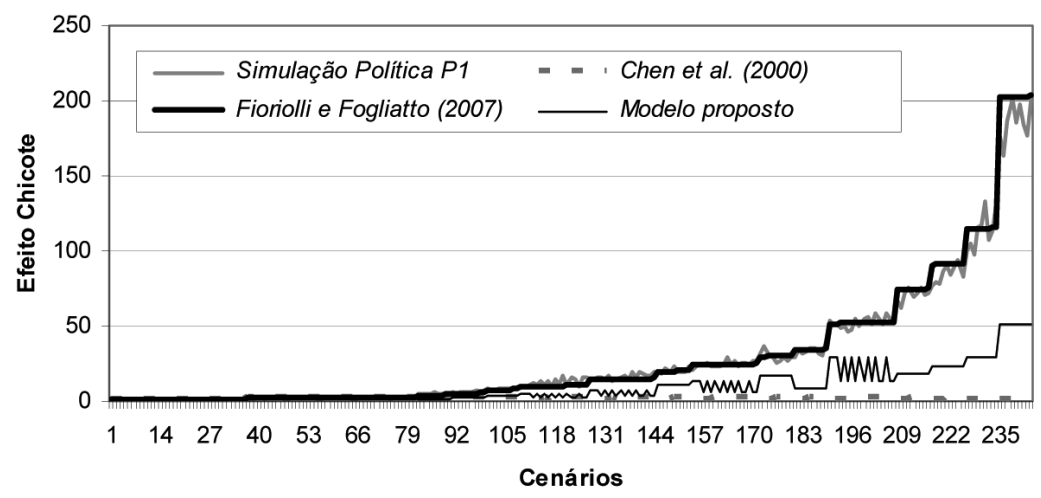

Figura 2: Simulação P1 versus modelos.

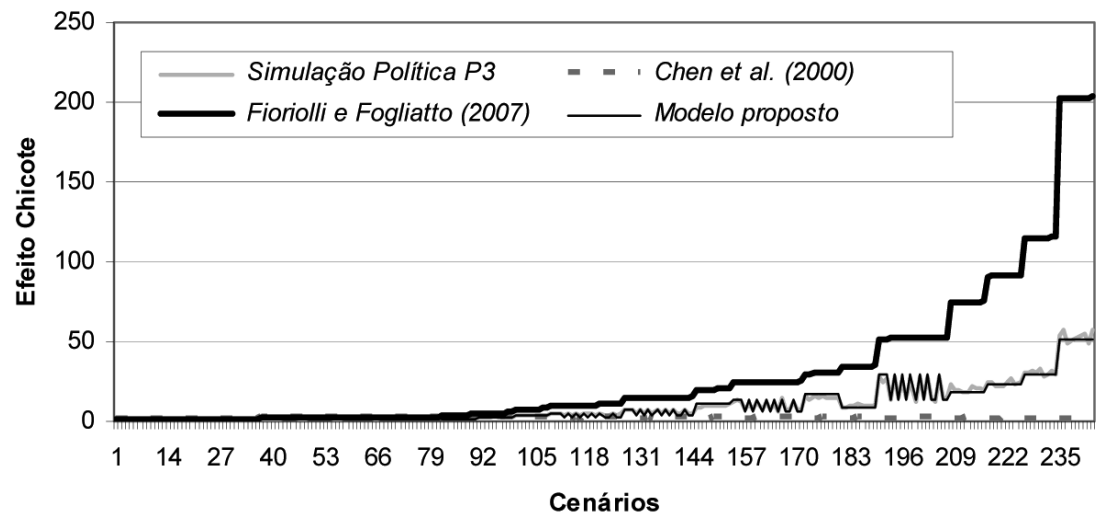

Figura 3: Simulação P3 versus modelos. 
não prevê ajustes no tamanho dos pedidos quando estes resultam negativos. O modelo proposto neste artigo, embora subestime o EC no caso de adoção da Política P1 (Figura 2), responde adequadamente à utilização da Política P3 (Figura 3). As diferenças observadas entre os resultados do modelo proposto e os valores simulados (Figura 2) podem ser consideradas não relevantes, na medida em que o aumento do desvio-padrão dos pedidos (gerado especificamente pela adoção da política P1) tende a amplificar desnecessariamente o EC.

Ao comparar as Figuras 2 e 3, observa-se que uma mudança de política de tratamento dos excessos de estoque pode reduzir o EC em 4 vezes nos cenários analisados.
Complementarmente, foram realizadas novas simulações (disponíveis no endereço http://rapidshare.com/ files/152105374/SIMULACAO_MODELOS.xls), considerando ambientes com lead time invariável. Os resultados dessas simulações são apresentados nas Figuras 4 e 5, relativos às Políticas $\mathrm{P} 1$ e $\mathrm{P} 3$, respectivamente.

Observa-se que o modelo de Chen et al. (2000), assim como o modelo de Fioriolli e Fogliatto (2007), apresenta excelente desempenho vinculado à Política P1 (Figura 4).

Estas constatações não surpreendem, uma vez que o pressuposto que caracteriza esta política (onde os excessos de estoque são devolvidos sem custo) está na base do desenvolvimento de ambos os modelos. No caso da adoção da

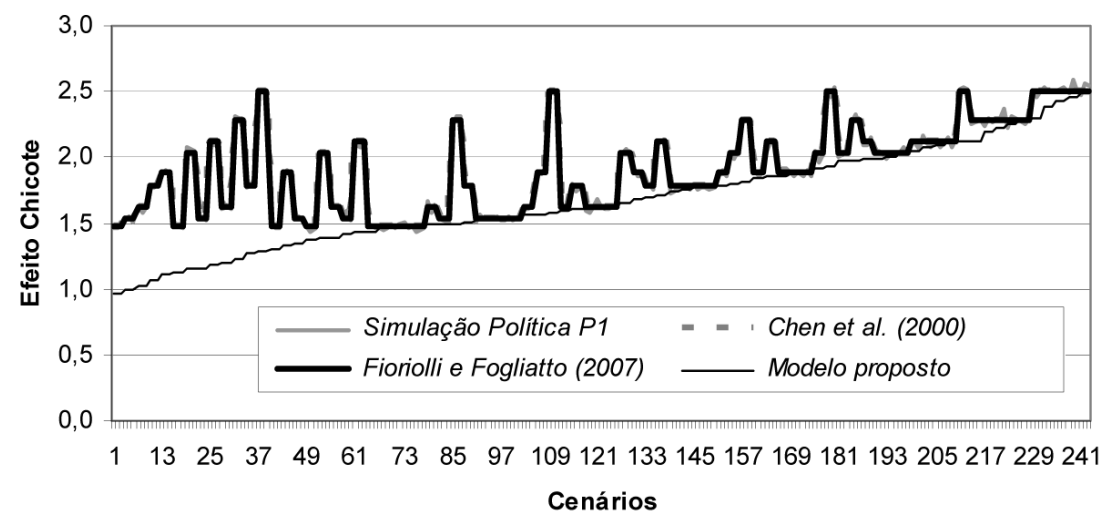

Figura 4: Simulação P1 versus modelos (lead time constante).

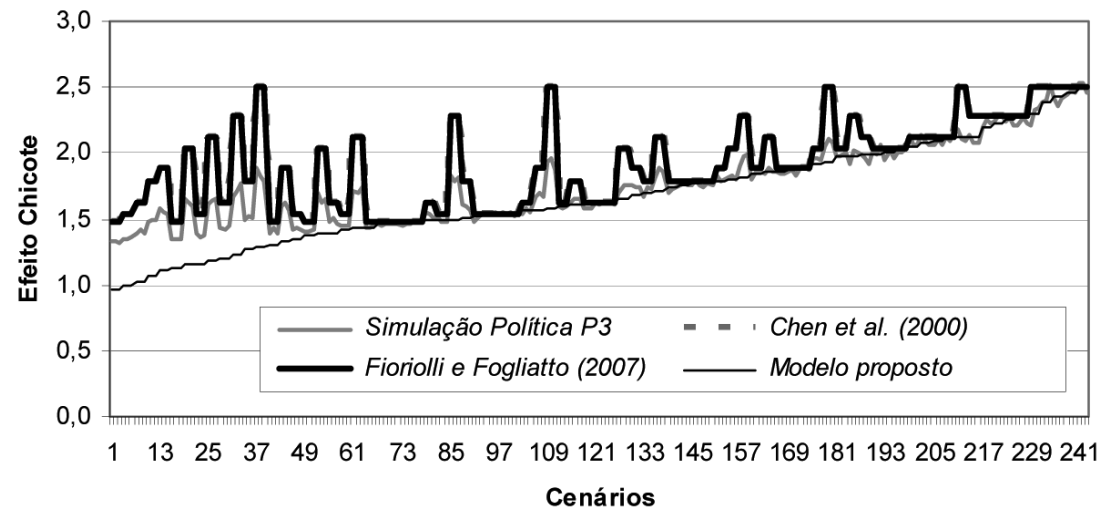

Figura 5: Simulação P3 versus modelos (lead time constante). 
Política P3, os melhores resultados são obtidos através do novo modelo, proposto na Seção 3 (ver Figura 5). De forma similar, este resultado também não surpreende porque o pressuposto que caracteriza a Política P3 (aproveitamento dos excessos de estoque no cálculo do tamanho dos pedidos dos períodos seguintes) constitui a base do desenvolvimento do modelo proposto nesta pesquisa.

A Tabela 5 apresenta um resumo das tendências primárias identificadas na análise de desempenho dos modelos, considerando as políticas de tratamento dos excessos de estoque e as características dos lead times.

Dado que a frequência de valores negativos obtidos no cálculo do tamanho dos pedidos é suficientemente alta para produzir distorções no dimensionamento do EC (mesmo em ambientes com lead time constante), torna-se necessário ajustar o tamanho dos pedidos. A Política P3, ao prever esses ajustes, ajuda a evitar aumentos na intensidade do EC, o que per se justifica a sua escolha na gestão de eventuais excessos de estoque em cadeias de abastecimento. Neste sentido, há elementos suficientes para considerar o novo modelo apresentado neste artigo como sendo mais preciso na quantificação do EC, frente aos modelos de Chen et al. (2000) e Fioriolli e Fogliatto (2007).

\section{CONCLUSÃO}

Com vistas a um dimensionamento mais preciso do EC, os resultados obtidos através da comparação de desempenho entre os modelos de Chen et al. (2000) e Fioriolli e Fogliatto (2007) evidenciam a importância da proposta apresentada neste artigo. A intensidade e o comportamento estocástico e serial do EC só podem ser adequadamente modelados se a variabilidade do lead time for incorporada ao processo de modelagem e se os excessos de estoque forem utilizados no cálculo do tamanho dos pedidos futuros.

Uma das consequências diretas do uso de lead times constantes nos modelos de quantificação do EC é o seu subdimensionamento (em alguns casos, apenas 5\% do fenômeno é quantificado). Por outro lado, conforme apresentado na Seção 4, o EC pode ter sua intensidade quadruplicada indevidamente, caso seja adotada uma política inadequada de tratamento dos excessos de estoque (rever Figuras 2 e 3 ).

O desempenho do novo modelo frente aos modelos de Chen et al. (2000) e Fioriolli e Fogliatto (2007), conforme apresentado na Tabela 5, combinado com a necessidade

\section{S resultados obtidos indicam que há expressivas diferenças de desempenho} entre os três modelos analisados. de adoção de uma política adequada para tratamento dos excessos de estoque (nos moldes da Política P3), permite sugerir que a modelagem proposta efetivamente contribui para dar maior precisão ao processo de quantificação do EC. Em razão da natureza deste fenômeno, simplificações tais como aquelas identificadas nos modelos analisados produzem distorções que, em função de sua magnitude, não podem ser desprezadas.

Com vistas ao aprimoramento do modelo ora proposto, novas pesquisas sobre a quantificação do EC podem ser desenvolvidas utilizando diferentes funções de distribuição de probabilidade para o lead time (por exemplo, gama ou lognormal). Adicionalmente, dado que a independência entre as variáveis lead time e demanda pode não se verificar em determinadas condições, sugere-se a incorporação da respectiva correlação nas futuras modelagens.

Tabela 5: Tendências primárias identificadas nas simulações.

\begin{tabular}{cccc}
\hline & Políticas e Modelos & $\sigma_{L}>0$ & $\sigma_{L}=0$ \\
\hline Política P1 & Chen et al. (2000) & Subestima & OK \\
& Fioriolli e Fogliatto (2007) & OK & SK \\
& Modelo proposto & Subestima & Superestima \\
\hline Política P3 & Chen et al. (2000) & Subestima & Superestima \\
& Fioriolli e Fogliatto (2007) & Superestima & OK \\
& Modelo proposto & OK & S
\end{tabular}




\section{Artigo recebido em 13/05/2008 Aprovado para publicação em 10/10/2008}

\section{REFERÊNCIAS}

ANDERSON JR., E. G.; FINE, C. H. Business cycles and productivity in capital equipment supply chains. In: Quantitative models for supply chain management. 6. ed. Boston: Kluwer Academic Publishers, 2003.

CARLSSON, C.; FULLÉR, R. A fuzzy approach to the bullwhip effect. In: Cybernetics and Systems 2000, Proceedings of the Fifteenth European Meeting on Cybernetics and Systems Research. Vienna, April 25-28, p. 228-233, 2000.

CHEN, F.; DREZNER, Z.; RYAN, J. K.; SIMCHILEVI, D. Quantifying the Bullwhip Effect in a Simple Supply Chain: The Impact of Forecasting, Lead Times, and Information. Management Science, v. 46, n. 3, p. 436-443, 2000.

The Bullwhip Effect: managerial insights on the impact of forecasting and information on variability in a supply chain.
In: Quantitative models for supply chain management. 6. ed. Boston: Kluwer Academic Publishers, 2003.

ELSAYED, E. A.; BOUCHER, T. O. Analysis and Control of Production Systems, 2. ed. New Jersey: Prentice Hall, 1994.

FIORIOLLI, J. C.; FOGLIATTO, F. S. Modelagem matemática do Efeito Chicote em ambientes com demanda e lead time estocásticos. In: XXXIX SBPO - Simpósio Brasileiro de Pesquisa Operacional, 2007, Fortaleza. Anais do XXXIX SBPO. Rio de Janeiro: Sobrapo, 2007.

FRANSOO, J. C.; WOUTERS, M. J. F. Measuring the Bullwhip Effect in the Supply Chain. Supply Chain Management, v. 5, n. 2, p. 78-89, 2000.
KAHN, J. Inventories and the volatility of production. American Economic Review, n. 77, p. 667-679, 1987.

LEE, H.; PADMANABHAN, V.; WHANG, S. Information Distortion in a Supply Chain: The Bullwhip Effect. Management Science, v. 43, n. 4, p. 546-558, 1997.

MOOD, A. M.; GRAYBILL, F. A.; BOES, D. C. Introduction to the theory of statistics. New York: McGraw-Hill, 1974.

SILVER, E. A.; PYKE, D. F.; PETERSON, R. Inventory management and production planning and scheduling, 3. ed. New York: John Wiley, 1998.

WARBURTON, R. D. H. An analytical Investigation of the Bullwhip Effect. Production and Operations Management, v. 13, n. 2, p. 150-160, 2004.

\section{AGRADECIMENTOS}

A pesquisa do Prof. Fogliatto é financiada pelo CNPq, através do processo PQ 301433/2005-4. Os autores gostariam de agradecer aos referees anônimos pela atenção dispensada ao artigo e pelas valiosas sugestões feitas visando a sua melhoria.

\section{SOBRE OS AUTORES}

José Carlos Fioriolli

Universidade Federal do Rio Grande do Sul

Escola de Administração

End.: Rua Washington Luis, 855 - 90010-460 - Porto Alegre/RS - Brasil

E-mail: jcfioriolli@ea.ufrgs.br

\section{Flávio Sanson Fogliatto}

Universidade Federal do Rio Grande do Sul

Programa de Pós-Graduação em Engenharia de Produção

End.: Av. Osvaldo Aranha, 99 - 5a andar - 90040-020 - Porto Alegre - RS

E-mail: ffogliatto@producao.ufrgs.br 